\title{
EDITORIAL
}

\section{Overview of the special issue on HBV immunity}

\author{
Antonio Bertoletti $^{1,2}$ and Fu-Sheng Wang ${ }^{3}$ \\ Cellular \& Molecular Immunology (2015) 12, 253-254; doi:10.1038/cmi.2015.24; published online 4 May 2015
}

Hepatitis B virus (HBV) has a unique relationship with humans. It is not only very successful in spreading amongst our species (a third of the human population has been in contact with the virus and approximately 200-300 million people are actively infected), ${ }^{1}$ but it has adapted to and co-evolved with us. This long-term relationship is demonstrated by the recent detection of hepadnavirus genomes in Mesozoic birds ${ }^{2}$ and by the estimation that HBV was already present in early humans at least 40, 000 years ago. ${ }^{3}$

The HBV co-evolution and adaptation with humans have affected the way that our immune system responds to this virus and can explain the primary characteristic of anti-HBV immunity: its subtle and often barely detectable nature in the infected subjects. Indeed, HBV is not highly immunogenic, and this virus is highly capable of avoiding or escaping immune detection rather than eliciting a strong immune reaction. Innate immune recognition, cytokine production, and HBVspecific T-cell responses can be detected in HBV-infected individuals, but often at levels lower than the levels observed in infections with other persistent human viruses such as

${ }^{1}$ Emerging infectious Diseases, Duke-NUS Medical School, Singapore, Singapore; ${ }^{2}$ Singapore Institute for Clinical Sciences, A*STAR, Singapore, Singapore and ${ }^{3}$ Research Center for Biological Therapy, The Institute of Translational Hepatology, Beijing, China

Correspondence: Antonio Bertoletti, Emerging Infectious Diseases, Duke-NUS Graduate Medical School, 8 College Road, Singapore 169857, Phone: +65 66011372.

E-mail: antonio@duke-nus.edu.sg ${ }^{1}$,Fu-Sheng Wang, Research Center for Biological Therapy, The Institute of Translational Hepatology, Beijing 302 Hospital, Beijing 100039, China.

E-mail: fswang302@163.com

Received: 24 February 2015; Revised: 25

February 2015; Accepted: 25 February 2015

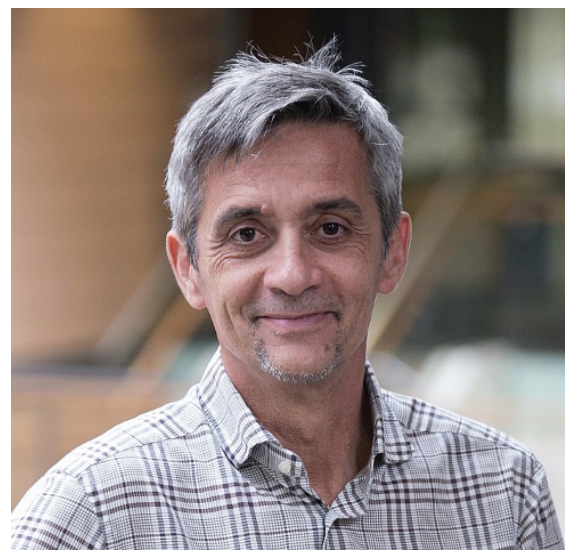

Dr. Antonio Bertoletti

Epstein-Barr virus, human immunodeficiency virus, or hepatitis $\mathrm{C}$ virus. ${ }^{4} \mathrm{HBV}$ is also not directly pathogenic to the host. HBV-related severe diseases are not directly caused by viral replication, but rather, are due to chronic immune-mediated inflammatory events that occur only in some actively infected subjects and often only present at an advanced age.

In this special issue of CMI dedicated to the discussion of immune response against HBV, we invited experts to summarize aspects of HBV immune pathogenesis that highlight this special relationship of $\mathrm{HBV}$ with human immunity.

In the first review "Contribution of toll-like receptors to the control of hepatitis $B$ virus infection by initiating antiviral innate responses and promoting specific adaptive immune responses," 5 Zhiyong Ma and colleagues addressed the complex interaction of HBV with the innate immune system, particularly with the toll-like receptors (TLR) system. The ability of HBV to evade and suppress the TLR-recognition system and the thera-

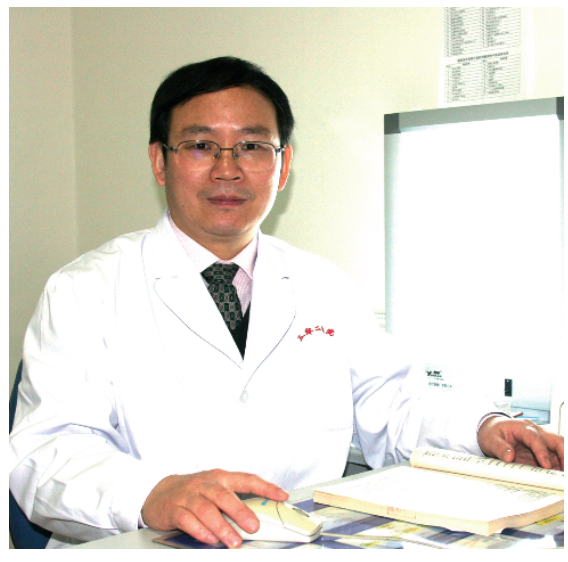

Dr. Fu-Sheng Wang

peutic potential of TLR agonists are discussed utilizing data derived from novel in vivo model systems.

The controversy regarding the impact of $\mathrm{HBV}$ on the functionality of the innate immune system is also the focus of a review by Adam J. Gehring and June Ann D’Angelo. In their chapter titled "Dissecting the dendritic cell controversy in chronic hepatitis B virus infection," 6 the authors summarize multiple studies investigating Dendritic cell (DC) function in chronic hepatitis $\mathrm{B}(\mathrm{CHB})$ patients to determine whether common observations can be drawn. DCs are central players in the induction of adaptive immunity, and the authors discuss data suggesting that dendritic cell defects are induced by HBV.

The relationship of $\mathrm{HBV}$ and innate immunity is also the focus of a review by Cheng Sun and colleagues. In their article "NK cell receptor imbalance and NK cell dysfunction in HBV infection and hepatocellular carcinoma,"7 the authors focused their attention not only on the functionality of 
natural killer (NK) cells during the early phases of HBV infection but particularly after the development of the most severe complication derived from HBV infection: hepatocellular carcinoma (HCC). The authors summarize data suggesting that, in $\mathrm{CHB}$, the progression to HCC is linked with a decrease in the frequency and functionality of intrahepatic NK cells. They also discussed the possibility of increasing NK cell function for therapeutic purposes.

The mechanisms and causes of liver damage during $\mathrm{CHB}$ infection in various infected subjects are the main focus of the following three distinct reviews by A Bertoletti and P. Kennedy, Roberto Aiolfi and Giovanni Sitia, and by Matteo Iannacone.

In the article "The immune tolerant phase of chronic $H B V$ infection: new perspectives on an old concept," 8 Bertoletti and Kennedy discussed the impact of the age of the infected host on the immune pathological process caused by HBV. The authors discussed the immunological significance of the concept of HBV immunotolerance during the early phases of infection, arguing that the modulation of liver disease during the life of HBVinfected individuals is driven by the physiological modifications of the inflammatory processes during aging and not by the often highly elusive CD8 T-cell response present in chronically infected patients.

Aiolfi and Sitia not only summarize the data that depicted the role of platelets in HBV infection in their article "Chronic hepatitis B: role of anti-platelet therapy in inflammation control" "but also introduce the concept of $\mathrm{CHB}$ as a primary inflammatory disease. The demonstration in animal models that the inhibition of platelet functionality can favorably modulate the extent of liver damage calls for an evaluation of this unconventional but simple anti-inflammatory therapeutic strategy in the clinical management of $\mathrm{CHB}$ patients.

CD8 $\mathrm{T}$ cells, the immune cell population with a pivotal role in the control and pathogenesis of HBV infection, is the focus of "Hepatic effector $C D 8^{+} T$-cell dynamics," ${ }^{10}$ a review by Matteo Iannacone. The author noted that "Understanding the signals that modulate the dynamics of $\mathrm{CD} 8^{+} \mathrm{T}$ cells in the liver is critical to gaining insight into the pathogenesis of acute and chronic HBV infection." This review presents new and interesting data that illustrate how $\mathrm{T}$ cells are trafficked in the liver and how they gain access to target cells, with a discussion on how technology can further increase our understanding of this important immunological process.

Finally, our review presents two articles that analyze components of the immune system that have been often overlooked in studies of HBV-specific immunity: T helpers and B-cell responses. In their review "Role of interleukin-21 in HBV infection: friend or foe?" 11 Yin Lin and colleagues recapitulate the present knowledge of the importance of the former factor in HBV infection, with a particular emphasis on the role of $\mathrm{T}$ helper follicular cells in HBV-infected individuals. The dual role of this cytokine in boosting the B-cellspecific antiviral response, or as a mediator of inflammation, was thoroughly discussed.

Lastly, due to the limited past knowledge of the B-cell response in HBV, Fu-Shen Wang and colleagues provided new findings that highlight the changes of B-cell functionality in CHB patients. ${ }^{12}$ Their article "Reversal of B-cell hyperactivation and functional impairment is associated with HBsAg seroconversion in chronic hepatitis B patients" provides long-awaited data on this aspect of human HBV immunity.

A cure for chronic hepatitis $\mathrm{C}$ has recently been achieved, leading to more attention and research focusing on HBV. The present therapeutic regimen for $\mathrm{CHB}$ patients involves antiviral treatments (using nucleotide analogs or interferon-alpha), which only suppress HBV replication without a clear effect on cccDNA and HBV antigens (especially HBsAg). It is difficult for the exhausted adaptive immunity present in $\mathrm{CHB}$ patients to play a role in complete control of the cccDNA and clearance of large amounts of HBsAg. Therefore, fully understanding the immune pathogenesis in these patients will help to promote the development of novel therapeutic approaches to boost antiviral immunity.

The aim of this special issue was certainly not to comprehensively cover all aspects of HBV immunity. Rather, we selected specific controversial areas of HBV immunity with the hope that by emphasizing the gaps in our knowledge, we can promote the generation of new ideas to cure CHB. We hope that this collection will offer an inspiration to all researchers fascinated by the complex relationship of HBV with its host.

1 Liaw YF, Chu CM. Hepatitis B virus infection. The Lancet 2009; 373: 582592.

2 Brosius JUR, Schmitz JUR, Kriegs JO, Suh A. The genome of a Mesozoic paleovirus reveals the evolution of hepatitis $B$ viruses. Nat Commun 2013; 4: 1791-1797.

3 Paraskevis D, Magiorkinis G, Magiorkinis E, Ho SYW, Belshaw R, Allain JP et al. Dating the origin and dispersal of hepatitis $B$ virus infection in humans and primates. Hepatology 2013; 57: 908-916.

4 Bertoletti A, Ferrari C. Innate and adaptive immune responses in chronic hepatitis $B$ virus infections: towards restoration of immune control of viral infection. Gut 2012; 61: 1754-1764.

5 Ma Z, Zhang E, Yang D, Lu M. Contribution of toll-like receptors to the control of hepatitis $B$ virus infection by initiating antiviral innate responses and promoting specific adaptive immune responses. Cell Mol Immunol 2015; 12: 273-282.

6 Gehring AJ, Ann D'Angelo J. Dissecting the dendritic cell controversy in chronic hepatitis $\mathrm{B}$ virus infection. Cell $\mathrm{Mol}$ Immunol 2015; 12: 283-291.

7 Sun C, Sun H, Zhang C, Tian Z. NK cell receptor imbalance and NK cell dysfunction in HBV infection and hepatocellular carcinoma. Cell Mol Immunol 2015; 12: 292-302.

8 Bertoletti A, Kennedy PT. The immune tolerant phase of chronic HBV infection: new perspectives on an old concept. Cell Mol Immunol 2015; 12: 258-263.

9 Aiolfi R, Sitia G. Chronic hepatitis B: role of anti-platelet therapy in inflammation control. Cell Mol Immunol 2015; 12: 264-268.

10 Iannacone M. Hepatic effector CD8(+) Tcell dynamics. Cell Mol Immunol 2015; 12: 269-272.

11 Li Y, Tang L, Hou J. Role of interleukin-21 in HBV infection: friend or foe? Cell Mol Immunol 2015; 12: 303-308.

12 Xu X, Zhang Z, Chen X, Nie W, Zou Z, Huang $A$ et al. Reversal of B-cell hyperactivation and functional impairment is associated with HBsAg seroconversion in chronic hepatitis B patients. Cell Mol Immunol 2015; 12: 309-316. 